\title{
Vibrational spectroscopy of neutral silicon clusters via far-IR-VUV two color ionization
}

\author{
André Fielicke, ${ }^{1, a)}$ Jonathan T. Lyon, ${ }^{1, b)}$ Marko Haertelt, ${ }^{1}$ Gerard Meijer, ${ }^{1}$ Pieterjan Claes, ${ }^{2}$ \\ Jorg de Haeck, ${ }^{2}$ and Peter Lievens ${ }^{2, c)}$ \\ ${ }_{1}^{1}$ Fritz-Haber-Institut der Max-Planck-Gesellschaft, Faradayweg 4-6, D-14195 Berlin, Germany \\ ${ }^{2}$ Laboratory of Solid State Physics and Magnetism and INPAC-Institute for Nanoscale Physics and Chemistry, \\ K. U. Leuven, Celestijnenlaan 200 D, B-3001 Leuven, Belgium
}

(Received 30 June 2009; accepted 20 October 2009; published online 5 November 2009)

\begin{abstract}
Tunable far-infrared-vacuum-ultraviolet two color ionization is used to obtain vibrational spectra of neutral silicon clusters in the gas phase. Upon excitation with tunable infrared light prior to irradiation with UV photons we observe strong enhancements in the mass spectrometric signal of specific cluster sizes. This allowed the recording of the infrared absorption spectra of $\mathrm{Si}_{6}, \mathrm{Si}_{7}$, and $\mathrm{Si}_{10}$. Structural assignments were made by comparison with calculated linear absorption spectra from quantum chemical theory. (C) 2009 American Institute of Physics. [doi:10.1063/1.3262803]
\end{abstract}

Silicon clusters have developed to a standard test system for the performance of search algorithms locating the global minimum of complex energy surfaces. ${ }^{1-3}$ Most of these calculations study neutral silicon clusters despite the lack of experimental information on their structures. So far, structure information from vibrational spectra has been obtained for neutral clusters up to $\mathrm{Si}_{7}$ only after their deposition and accumulation in cryogenic matrices. ${ }^{4-6}$ Gas-phase studies on neutral Si clusters include measurements of ionization potentials, ${ }^{7}$ of optical absorption spectra, ${ }^{8}$ and of bond energies by Knudsen cell mass spectrometry. ${ }^{9}$ The structures of charged silicon clusters are much better investigated. For example, cross sections of $\mathrm{Si}_{n}^{+/-}$have been determined from ion mobility measurements. ${ }^{10,11}$ Anionic silicon clusters have been intensively investigated by photoelectron spectroscopy ${ }^{12-16}$ and for a few small clusters this has allowed to resolve vibrational progressions. ${ }^{17-19}$ Recently we have reported the measurement of infrared spectra for $\mathrm{Si}_{6-21}^{+}$ with IR multiple photon dissociation using the evaporation of weakly bound $\mathrm{Xe}$ atoms as messenger for the absorption. ${ }^{20}$ As the vibrational fingerprint is particularly sensitive to the geometrical structure, this led for $\mathrm{Si}_{8}^{+}$to the assignment of an edge-capped pentagonal bipyramid structure that was previously not considered. For larger clusters the change in the structural motifs from pentagonal bipyramids to tricapped trigonal prisms has been confirmed. In a similar way, structural information on cationic transition metal doped silicon clusters has been obtained using Ar atoms as a messenger. ${ }^{21}$

Although the messenger technique has been recently applied very successfully for obtaining infrared spectra of strongly bound gas-phase clusters, ${ }^{22}$ it has the inherent disadvantage of possible perturbations of the cluster. ${ }^{23,24}$ Interaction with the messenger may even alter the relative ener-

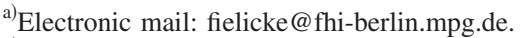

${ }^{b)}$ Present address: Department of Natural Sciences, Clayton State University, 2000 Clayton State Blvd., Morrow, GA 30260, USA.

${ }^{c}$ Electronic mail: peter.lievens@fys.kuleuven.be.
}

getic order of cluster isomers. ${ }^{25}$ In addition, forming a complex with a weakly bound messenger often becomes difficult for neutral species. For instance, for gold clusters complex formation with $\mathrm{Kr}$ atoms has been observed only at $\approx 100 \mathrm{~K}$, whereas atoms of lighter rare gases do not bind. However, the attachment of the highly polarizable $\mathrm{Kr}$ atoms is found to affect the vibrational spectra of the Au clusters. ${ }^{26}$ A method that reveals the absorption of IR photons by clusters without the need to form a messenger complex is therefore highly desirable. IR resonance enhanced multiple photon ionization (IR-REMPI) (Ref. 27) is such a technique that does not require formation of a messenger complex. IRREMPI relies on the subsequent absorption of a large number of IR photons by a single cluster followed by delayed thermionic ionization. This merely is the case if the cluster is very stable and does not dissociate beforehand. This is fulfilled, however, only for a few types of clusters, mainly from refractory materials such as some transition metals, e.g., $\mathrm{Nb}$ or $\mathrm{W}$, several metal oxides and carbides, as well as fullerenes. ${ }^{28}$

More generally applicable is the combination of IR excitation with near threshold photoionization. This two color ionization scheme relies on the absorption of a single or few IR photons prior to interaction with a UV photon to lift the total internal energy of the species above the ionization threshold. The direct photoionization generally prevails over the slower statistical fragmentation processes. The formed ions can be sensitively detected by means of mass spectrometry. By scanning the energy of the IR photons the ionization efficiency changes and the recorded ion intensity reflects the IR absorption spectrum of the corresponding neutral species. The high sensitivity of such an approach has been demonstrated for para-amino benzoic acid using a free electron laser as a tunable radiation source in the midinfrared range, where other intense lasers are lacking. ${ }^{29}$ We here apply such an IR-UV two color ionization scheme for obtaining the vibrational spectra of neutral silicon clusters in the far-IR range between 225 and $550 \mathrm{~cm}^{-1}$. These results constitute 


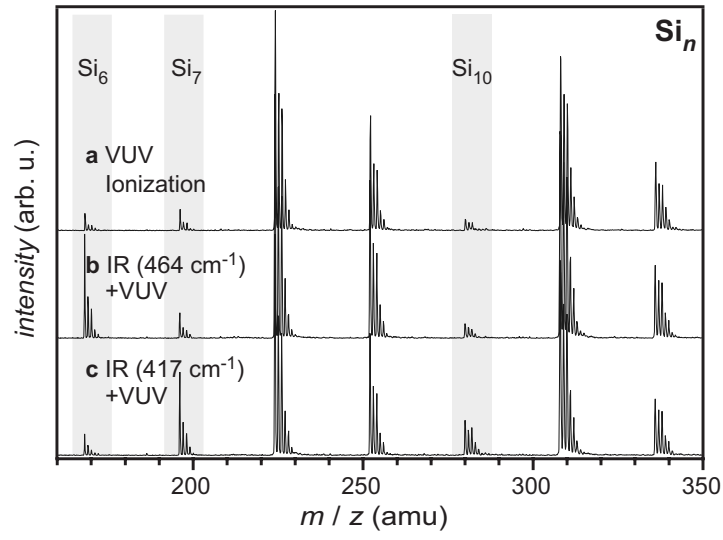

FIG. 1. (a) Mass spectra of silicon clusters obtained upon ionization with $7.87 \mathrm{eV}$ VUV photons. (b) Initial irradiation of the neutral cluster distribution with intense IR light at $464 \mathrm{~cm}^{-1}$ leads to a sixfold increase in the signal of $\mathrm{Si}_{6}^{+}$, while the remaining mass spectrum is unchanged. (c) Upon irradiation with infrared light of $417 \mathrm{~cm}^{-1}$ enhancement of the intensities of $\mathrm{Si}_{7}^{+}$and $\mathrm{Si}_{10}^{+}$is observed.

the first vibrational spectra of isolated neutral silicon clusters in the gas phase.

Si clusters are produced as described before for the cationic species ${ }^{20}$ via laser ablation in a source capable of producing also binary clusters by coablation from two different targets $^{30}$ in a rare-gas atmosphere. The clusters are thermalized to $100 \mathrm{~K}$ in a cooled extension channel of the source. The cluster distribution is frozen out by expansion into vacuum and the formed molecular beam is shaped by a skimmer and an aperture with $1 \mathrm{~mm}$ opening. The neutral clusters are ionized by a weakly focused beam from an $\mathrm{F}_{2}$ laser emitting vacuum-ultraviolet (VUV) photons of $7.87 \mathrm{eV}$ energy. The resulting cationic distribution is analyzed in a reflectron time-of-flight mass spectrometer. Care has been taken to reduce the photon fluence to an extent that the cluster distribution [Fig. 1(a)] resembles the single photon ionization mass spectrum. ${ }^{31}$ Under these conditions intense signals for $\mathrm{Si}_{8,9, n \geq 11}$ are obtained. $\mathrm{Si}_{6,7,10}$ show rather low intensity, while smaller clusters $(n \leq 5)$ are essentially not observed. This corresponds to the known cluster size specific ionization energies, which are clearly below $7.87 \mathrm{eV}$ for $\mathrm{Si}_{8,9, n \geq 11}$, distinctly higher than $7.97 \mathrm{eV}$ for $\mathrm{Si}_{1-5}$, but within 7.87-7.97 $\mathrm{eV}$ for $\mathrm{Si}_{6,7,10} \cdot{ }^{7}$ We observe that a small amount of $\mathrm{Si}_{6,7,10}$ is always ionized by the $\mathrm{F}_{2}$ laser light. This may be attributed either to a hot fraction of the clusters, or to absorption of multiple VUV photons.

The relative mass spectrometric intensities can change, however, if the cluster beam is irradiated with IR light before interaction with the VUV radiation ( $b$ and $c$ in Fig. 1). In these experiments, a beam of intense pulsed IR radiation emitted from the Free Electron Laser for Infrared eXperiments (FELIX) ${ }^{32}$ is counterpropagating the molecular beam and focused onto the aperture shaping the cluster beam. This ensures that all clusters detected afterwards passed the focal range and interacted with the IR light. The IR pulse energies were about $20-40 \mathrm{~mJ}$ at a pulse length of $\approx 5 \mu \mathrm{s}$. If the frequency of the IR light is in resonance with an IR active mode of the cluster it can absorb one or more IR photons. Subsequent internal vibrational energy redistribution leads to

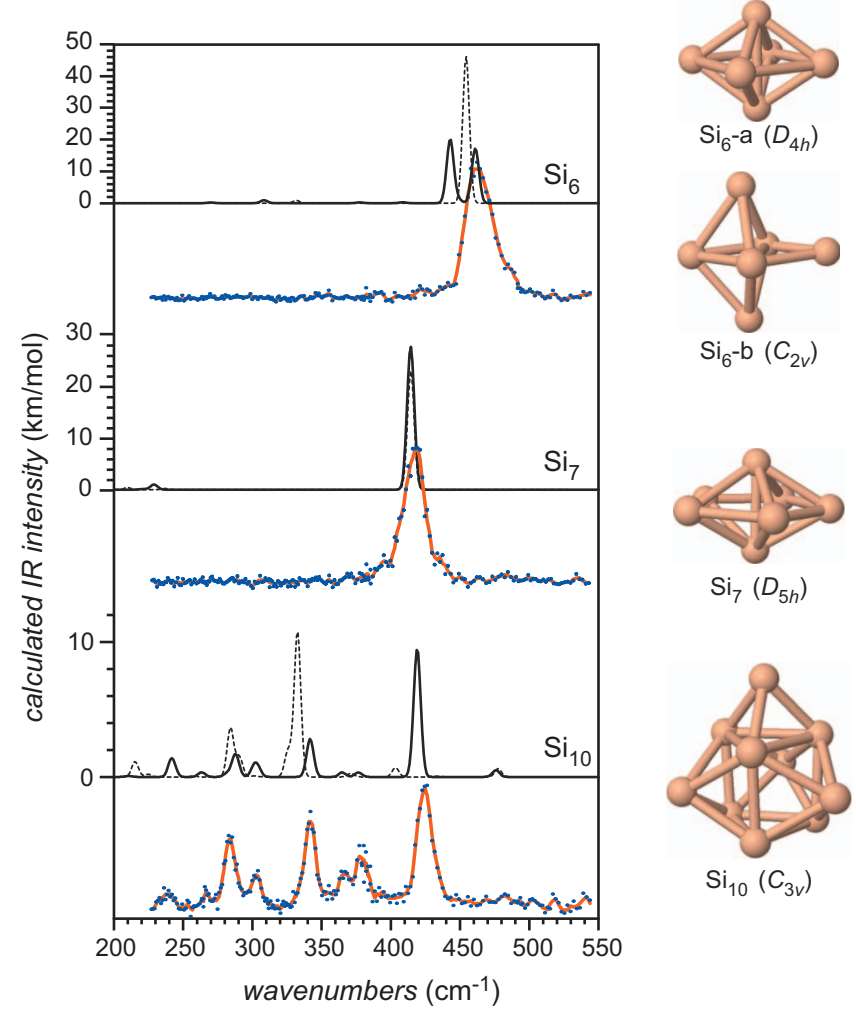

FIG. 2. Comparison of experimental IR spectra for $\mathrm{Si}_{6,7,10}$ with predictions from theory. The experimental spectra are assembled from data obtained in three independent runs similarly as described before (Ref. 20). The (blue) dots are the resulting original data points, while the (red) lines correspond to a three-point adjacent average. Experimental absorption cross sections are given on a linear scale in arbitrary units. The solid lines in the calculated spectra represent the results from DFT, while the dashed lines correspond to the MP2 calculations. Calculated frequencies are linearly scaled by a factor of 1.03 (DFT) (Table I) and 0.96 (MP2), respectively, and the stick spectra are folded with a Gaussian line width function of $5 \mathrm{~cm}^{-1}$ full width at half maximum.

a thermal heating of the cluster. The increase in internal energy can enhance the ionization efficiency upon interaction with VUV photons as it may raise the total energy (higher) above the ionization threshold. The enhancement is a purely thermal effect as the clusters are irradiated with IR light $\approx 30 \mu$ s before interaction with the VUV photons. The traces $\mathrm{b}$ and $\mathrm{c}$ in Fig. 1 demonstrate the change in the ionization efficiencies upon pumping the neutral cluster distribution with 464 and $417 \mathrm{~cm}^{-1}$ photons, respectively, leading to strong signal enhancements for $\mathrm{Si}_{6}^{+}$in the first case, and for $\mathrm{Si}_{7}^{+}$as well as $\mathrm{Si}_{10}^{+}$in the second.

Recording the signal enhancements as a function of the IR frequency yields the IR absorption spectra of the neutral clusters. Figure 2 shows for $\mathrm{Si}_{6,7,10}$ the relative enhancements of the ion signal $\left(I-I_{0}\right) / I_{0}$ normalized by the IR laser fluence recorded in the $225-550 \mathrm{~cm}^{-1}$ range. The IR spectra of $\mathrm{Si}_{6}$ and $\mathrm{Si}_{7}$ are dominated by a single peak at $464 \pm 1$ and $417 \pm 1 \mathrm{~cm}^{-1}$, respectively, while the spectrum of $\mathrm{Si}_{10}$ is clearly more complex.

For $\mathrm{Si}_{6}$ and $\mathrm{Si}_{7}$ these findings can be compared with previous experimental results. In matrix isolation experiments using different rare gas hosts, a single IR absorption band found at $462.9 \mathrm{~cm}^{-1}$ in $\mathrm{Ne}, 460.9 \mathrm{~cm}^{-1}$ in $\mathrm{Ar}$, and $458.5 \mathrm{~cm}^{-1}$ in $\mathrm{Kr}$ has been assigned to the $e_{u}$ mode of $\mathrm{Si}_{6}$ in 
TABLE I. Assignments of experimentally observed IR bands and comparison with values calculated by DFT (frequencies scaled by a factor of 1.03) The experimental frequencies have an estimated uncertainty of $\pm 1 \mathrm{~cm}^{-1}$.

\begin{tabular}{lcccc}
\hline \hline Cluster & Symmetry & $\begin{array}{c}\nu_{\text {expt }} \\
\left(\mathrm{cm}^{-1}\right)\end{array}$ & $\begin{array}{c}\nu_{\text {calc }} \\
\left(\mathrm{cm}^{-1}\right)\end{array}$ & Mode \\
\hline $\mathrm{Si}_{6}{ }^{\mathrm{a}}$ & $D_{4 h}$ & 464 & 453.9 & $e_{u}$ \\
$\mathrm{Si}_{7}$ & $D_{5 h}$ & 417 & 413.9 & $e_{1}^{\prime}$ \\
$\mathrm{Si}_{10}$ & $C_{3 v}$ & 425 & 418.6 & $e$ \\
& 380 & 376.0 & $e$ \\
& 366 & 364.3 & $a_{1}$ \\
& 342 & 341.0 & $a_{1}$ \\
& 303 & $301.0,303.8$ & $e, a_{1}$ \\
& 284 & 287.0 & $e$ \\
& 267 & 262.5 & $a_{1}$ \\
& 239 & 241.0 & $e$ \\
\hline
\end{tabular}

${ }^{\mathrm{a}}$ Theoretical data from MP2 calculations scaled by 0.96 .

the $D_{4 h}$ structure (see Fig. 2), while a band at $422.4 \mathrm{~cm}^{-1}$ (Ar) and $420.4 \mathrm{~cm}^{-1}(\mathrm{Kr})$ is assigned to the $e_{1}^{\prime}$ mode of $D_{5 h}$ $\mathrm{Si}_{7} .{ }^{6}$ Analysis of the vibrational progressions in the photoelectron spectrum of $\mathrm{Si}_{7}^{-}$revealed a frequency of $385 \pm 20 \mathrm{~cm}^{-1}$ for neutral $\mathrm{Si}_{7} \cdot{ }^{18,19}$ This has been compared with the data from Raman spectroscopy measured in an $\mathrm{N}_{2}$ matrix. In that range $\mathrm{Si}_{7}$ has a Raman active mode at $435 \mathrm{~cm}^{-1}\left(a_{1}^{\prime}\right)^{4,5}$ The deviation between the frequencies obtained from the photoelectron spectrum and in the matrix isolation experiment has been suggested to be due to matrix effects. A comparison of the here reported frequencies of the IR active modes of the gas-phase clusters with those from the matrix experiments suggests, however, that there are only very minor shifts.

The experimental spectra in Fig. 2 are compared with the results of calculations by using density functional theory (DFT) and second-order Møller-Plesset perturbation theory (MP2) performed within GAUSSIAN 03. ${ }^{33}$ Details on the calculations can be found in Ref. 20 and in the supplementary information. ${ }^{34}$ The structures of the cationic clusters have been used as starting geometries in these calculations. In general, good agreement between the experimental and the DFT results is obtained, which indicates identification of the correct structures. Good predictive capabilities of our DFT approach also have been found for the cationic Si clusters. MP2 calculations give a better representation of the spectrum of $\mathrm{Si}_{6}$, while for $\mathrm{Si}_{7}$ the performance is nearly identical to the DFT approach. For $\mathrm{Si}_{10}$ the results from MP2 calculations agree to a lesser extent with the experiment although the structure is nearly unchanged with respect to the DFT predictions.

The reason for the differences between the spectra of $\mathrm{Si}_{6}$ predicted by either DFT or MP2 is that depending on the method, different lowest energy structures are determined for $\mathrm{Si}_{6} \cdot{ }^{4,35-37}$ DFT usually favors an edge-capped trigonal prism of $C_{2 v}$ symmetry, while within MP2 the distorted octahedron $\left(D_{4 h}\right)$ is found as the minimum energy structure. Higher level methods taking electron correlation and configurational interactions into account reveal that the potential surface around the ground state is very flat and the structure fluctuates between the distorted octahedron, the edge-capped trigonal prism, and the face-capped trigonal prism. ${ }^{36}$ Lately, this has been explained by $\mathrm{Si}_{6}$ undergoing a pseudo Jahn-Teller distortion and it has been shown that the predictive capabilities of the theoretical methods depend on their treatment of the pseudo Jahn-Teller effect. ${ }^{37}$ Within our DFT calculations $\mathrm{Si}_{6}$ is found in a ${ }^{1} A_{1}$ state with $C_{2 v}$ symmetry and MP2 predicts the distorted octahedron $\left(D_{4 h}\right)$ in the ${ }^{1} A_{1 g}$ state. The experimental finding is consistent with either of them as the band splitting predicted for the $C_{2 v}$ structure might not be resolved in the experiment. Anyhow, the band observed for $\mathrm{Si}_{6}$ is noticeably broader in comparison with those observed for the other sizes. $\mathrm{Si}_{7}$ is a pentagonal bipyramid with ${ }^{1} A_{1}^{\prime}$ electronic configuration and the structure of $\mathrm{Si}_{10}$ we identify as fourfold capped trigonal prism in a ${ }^{1} A_{1}$ state, which is in agreement with previous predictions. ${ }^{10,38,39}$ Other isomers of $\mathrm{Si}_{10}$ are at least $0.56 \mathrm{eV}$ higher in energy and exhibit rather different vibrational spectra (see supporting information ${ }^{34}$ ). For these three neutral silicon clusters the structures are very similar to their cationic and anionic counterparts.

In summary, we present vibrational spectra of small neutral silicon clusters obtained via tunable IR-VUV two photon ionization. This technique allows for characterization of the cluster structures without the need of a possibly perturbing messenger or a host matrix. As many strongly bound clusters have their ionization energies in a range accessible by UV lasers and near threshold ionization can be easily achieved, it is expected that tunable IR-UV two photon ionization becomes a more generally applicable method for the investigation of vibrational spectra of neutral clusters.

We gratefully acknowledge the support of the Stichting voor Fundamenteel Onderzoek der Materie (FOM) in providing beam time on FELIX. The authors thank the FELIX staff for their skillful assistance, in particular Dr. B. Redlich and Dr. A.F.G. van der Meer. This work is supported by the Fund for Scientific Research-Flanders (FWO), the Flemish Concerted Action (Contract No. GOA/2009/06), and the Belgian Interuniversity Poles of Attraction (Grant No. IAP/P5/01) programs and by the Cluster of Excellence "Unifying Concepts in Catalysis" coordinated by the Technische Universität Berlin and funded by the Deutsche Forschungsgemeinschaft. J.T.L. thanks the Alexander von Humboldt Foundation and P.C., the Institute for the Promotion of Innovation by Science and Technology in Flanders (IWT) for financial support.

${ }^{1}$ U. Röthlisberger, W. Andreoni, and M. Parrinello, Phys. Rev. Lett. 72, 665 (1994).

${ }^{2}$ A. Tekin and B. Hartke, J. Theor. Comput. Chem. 4, 1119 (2005).

${ }^{3}$ S. Goedecker, W. Hellmann, and T. Lenosky, Phys. Rev. Lett. 95, 055501 (2005).

${ }^{4}$ E. C. Honea, A. Ogura, C. A. Murray, K. Raghavachari, W. O. Sprenger, M. F. Jarrold, and W. L. Brown, Nature (London) 366, 42 (1993).

${ }^{5}$ E. C. Honea, A. Ogura, D. R. Peale, C. Felix, C. A. Murray, K. Raghavachari, W. O. Sprenger, M. F. Jarrold, and W. L. Brown, J. Chem. Phys. 110, 12161 (1999)

${ }^{6} \mathrm{~S}$. Li, R. J. Van Zee, J. W. Weltner, and K. Raghavachari, Chem. Phys. Lett. 243, 275 (1995).

${ }^{7}$ K. Fuke, K. Tsukamoto, F. Misaizu, and M. Sanekata, J. Chem. Phys. 99, 7807 (1993)

${ }^{8}$ K. D. Rinnen and M. L. Mandich, Phys. Rev. Lett. 69, 1823 (1992).

${ }^{9}$ G. Meloni and K. A. Gingerich, J. Chem. Phys. 115, 5470 (2001).

${ }^{10}$ K.-M. Ho, A. A. Shvartsburg, B. Pan, Z.-Y. Lu, C.-Z. Wang, J. G. Wacker, J. L. Fye, and M. F. Jarrold, Nature (London) 392, 582 (1998). 
${ }^{11}$ A. A. Shvartsburg, R. R. Hudgins, P. Dugourd, and M. F. Jarrold, Chem. Soc. Rev. 30, 26 (2001).

${ }^{12}$ O. Cheshnovsky, S. H. Yang, C. L. Pettiette, M. J. Craycraft, Y. Liu, and R. E. Smalley, Chem. Phys. Lett. 138, 119 (1987).

${ }^{13}$ J. Müller, B. Liu, A. A. Shvartsburg, S. Ogut, J. R. Chelikowsky, K. W. M. Siu, K.-M. Ho, and G. Gantefor, Phys. Rev. Lett. 85, 1666 (2000).

${ }^{14}$ M. Astruc Hoffmann, G. Wrigge, B. v. Issendorff, J. Müller, G. Ganteför, and H. Haberland, Eur. Phys. J. D 16, 9 (2001).

${ }^{15}$ G. Meloni, M. J. Ferguson, S. M. Sheehan, and D. M. Neumark, Chem. Phys. Lett. 399, 389 (2004).

${ }^{16}$ J. Bai, L.-F. Cui, J. Wang, S. Yoo, X. Li, J. Jellinek, C. Koehler, T Frauenheim, L.-S. Wang, and X. C. Zeng, J. Phys. Chem. A 110, 908 (2006).

${ }^{17}$ T. N. Kitsopoulos, C. J. Chick, A. Weaver, and D. M. Neumark, J. Chem. Phys. 93, 6108 (1990).

${ }^{18}$ G. S. Icking-Konert, H. Handschuh, P. S. Bechthold, G. Ganteför, B. Kessler, and W. Eberhardt, Surf. Rev. Lett. 3, 483 (1996).

${ }^{19}$ C. Xu, T. R. Taylor, G. R. Burton, and D. M. Neumark, J. Chem. Phys. 108, 1395 (1998).

${ }^{20}$ J. T. Lyon, P. Gruene, A. Fielicke, G. Meijer, E. Janssens, P. Claes, and P. Lievens, J. Am. Chem. Soc. 131, 1115 (2009).

${ }^{21}$ P. Gruene, A. Fielicke, G. Meijer, E. Janssens, V. T. Ngan, M. T. Nguyen, and P. Lievens, ChemPhysChem 9, 703 (2008).

${ }^{22}$ K. R. Asmis, A. Fielicke, G. von Helden, and G. Meijer, in Atomic Clusters: From Gas Phase to Deposited, edited by D. P. Woodruff (Elsevier, Amsterdam, 2007), p. 327.

${ }^{23}$ M. B. Knickelbein, J. Chem. Phys. 100, 4729 (1994).
${ }^{24}$ R. Gehrke, P. Gruene, A. Fielicke, G. Meijer, and K. Reuter, J. Chem. Phys. 130, 034306 (2009).

${ }^{25}$ K. R. Asmis and J. Sauer, Mass Spectrom. Rev. 26, 542 (2007).

${ }^{26}$ P. Gruene, D. M. Rayner, B. Redlich, A. F. G. van der Meer, J. T. Lyon, G. Meijer, and A. Fielicke, Science 321, 674 (2008).

${ }^{27}$ G. von Helden, I. Holleman, G. M. H. Knippels, A. F. G. van der Meer, and G. Meijer, Phys. Rev. Lett. 79, 5234 (1997).

${ }^{28}$ G. von Helden, D. van Heijnsbergen, and G. Meijer, J. Phys. Chem. A 107, 1671 (2003)

${ }^{29}$ M. Putter, G. von Helden, and G. Meijer, Chem. Phys. Lett. 258, 118 (1996).

${ }^{30}$ W. Bouwen, P. Thoen, F. Vanhoutte, S. Bouckaert, F. Despa, H. Weidele, R. E. Silverans, and P. Lievens, Rev. Sci. Instrum. 71, 54 (2000).

${ }^{31}$ D. J. Trevor, D. M. Cox, K. C. Reichmann, R. O. Brickman, and A. Kaldor, J. Phys. Chem. 91, 2598 (1987).

${ }^{32}$ D. Oepts, A. F. G. van der Meer, and P. W. van Amersfoort, Infrared Phys. Technol. 36, 297 (1995).

${ }^{33}$ M. J. Frisch, G. W. Trucks, H. B. Schlegel et al., GAUSSIAN 03, Revision C.02, Gaussian, Inc., Wallingford, CT, 2004.

${ }^{34}$ See EPAPS supplementary material at http://dx.doi.org/10.1063/ 1.3262803 for details of the quantum chemical calculations.

${ }^{35}$ C. Zhao and K. Balasubramanian, J. Chem. Phys. 116, 3690 (2002).

${ }^{36}$ A. D. Zdetsis, J. Chem. Phys. 127, 014314 (2007).

${ }^{37}$ P. Karamanis, D. Zhang-Negrerie, and C. Pouchan, Chem. Phys. 331, 417 (2007).

${ }^{38}$ K. Raghavachari and C. M. Rohlfing, Chem. Phys. Lett. 198, 521 (1992).

${ }^{39}$ X. Zhu and X. C. Zeng, J. Chem. Phys. 118, 3558 (2003). 